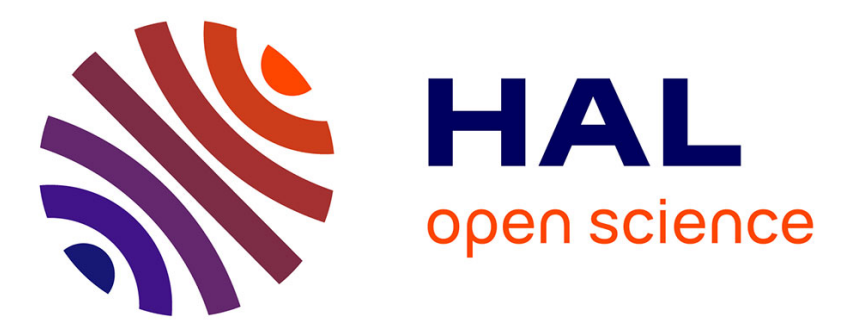

\title{
Le service de santé militaire dans les intendances de Flandre et de Hainaut : de la liberté provinciale à l'uniformisation nationale
}

Renaud Limelette

\section{- To cite this version:}

Renaud Limelette. Le service de santé militaire dans les intendances de Flandre et de Hainaut : de la liberté provinciale à l'uniformisation nationale. La construction des professions juridiques et médicales. Europe occidentale, XVIIIe-XXe siècle, Presses universitaires du Septentrion, 2020, 978-2-7574-2959-4. hal-02967832

\section{HAL Id: hal-02967832 \\ https://hal.univ-lille.fr/hal-02967832}

Submitted on 15 Oct 2020

HAL is a multi-disciplinary open access archive for the deposit and dissemination of scientific research documents, whether they are published or not. The documents may come from teaching and research institutions in France or abroad, or from public or private research centers.
L'archive ouverte pluridisciplinaire HAL, est destinée au dépôt et à la diffusion de documents scientifiques de niveau recherche, publiés ou non, émanant des établissements d'enseignement et de recherche français ou étrangers, des laboratoires publics ou privés. 


\section{Le service de santé militaire dans les intendances de Flandre et de Hainaut : de la liberté provinciale à l'uniformisation nationale}

Renaud Limelette

Dans le Corpus iuris civilis, quelques passages indiquent que l'assistance aux militaires était pratiquée dans l'Empire romain. Des fragments du Digeste relatent que les chefs militaires avaient parmi leurs devoirs celui d'inspecter les malades ${ }^{1}$ et que les médecins des hôpitaux militaires étaient exempts de charges publiques $^{2}$ en raison de la pénibilité de leur emploi. Ainsi, dans les légions impériales, l’assistance aux militaires dépendait du préfet du camp qui organisait le valetudinarium ${ }^{3}$, nom donné à l'hôpital militaire. Il était secondé par le medicus castrensis, dont le rôle était de coordonner l'action des médecins de la légion. Les soins étaient pratiqués au niveau de la cohorte par les medici cohortis. Déjà, des praticiens étaient spécialisés, comme les medici chirurgicos en chirurgie et les medici ocularis en ophtalmologie.

Au Moyen Âge, un service de santé militaire organisé, tel celui des légions romaines, n’existe pas. Certes, sous le règne de Philippe le Bel apparaissent quelques médecins et chirurgiens barbiers ${ }^{4}$, mais ce sont des praticiens au service d'un prince, comme Henri de Mondeville ${ }^{5}$, chirurgien de Philippe le Bel, qui accompagna le roi en Flandre en 1297, puis Charles de Valois à Courtrai et à la bataille de Mons-en-Pévèle. Pour remédier à l'absence de soins organisés, le soldat reçoit néanmoins une solde pour subvenir à ses frais de subsistance, d'habillement, d'armement et de traitement en cas de blessure ou de maladie ${ }^{6}$. Ce n'est qu'au $\mathrm{XVI}^{\mathrm{e}}$ siècle que le pouvoir royal prend en considération le sort du soldat malade ou blessé. D'abord timidement : un règlement du 20 janvier 1514 sur le service des gendarmes prescrit

1 D. 49. 16. 12, Macer lib. 1, De re militari. Aemilius Macer était un juriste romain qui vivait sous le règne d'Alexandre Sévère; plusieurs fragments du Digeste sont extraits de ses œuvres, dont De re militari, De officio praesidis, De publicus judiciis. Pour une notice biographique plus complète, voir William SMITH, A Dictionary of Greek and Roman Biography and Mythology, Londres, J. Murray, 1880, t. 2, p. 883.

2 D. 50. 6. 6, Taruntenus Paternus lib. 1 Militarium. Taruntenus Paternus vécut sous l'empereur Commode; deux fragments de son Militarium sont inscrits dans le Digeste. Pour une notice biographique plus complète, voir SMITH, A Dictionary of Greek and Roman Biography..., op. cit., t. 3, p. 135.

3 André Pichот, « La santé et la vie », Philosophia Scientice [En ligne], 12-2 | 2008, mis en ligne le 01 octobre 2011. URL : http://journals.openedition.org/philosophiascientiae/101; DOI : 10.4000/philosophiascientiae.10.

4 Jean Des Cilleuls, J. Pesme, Jean Hassenforder et G. Hugonot, « Le Service de santé militaire de ses origines à nos jours », Revue internationale des services de santé des armées de terre, de mer et de l'air, nº hors série, 1961, p. 5.

5 Édouard NICAISE, Chirurgie de maître Henri de Mondeville, chirurgien de Philippe Le Bel roi de France, composée de 1306 à 1320, Paris, G. Baillière, 1893 ; Valérie AlvarEz-CATALA, « Henri de Mondeville ou la vie d'un maître en chirurgie de Montpellier dans le contexte médical et religieux du XIV siècle », Thèse de médecine, Montpellier I, 1993.

6 Adolphe GauldreE-Boileau, L'administration militaire dans les temps modernes, Paris, J. Dumaine, 1871, p. 65. 
que le malade absent lors de la revue doit pourtant être inscrit au rôle ${ }^{7}$, de sorte qu'il reçoit sa solde malgré son état. La même mesure est mentionnée dans l'édit de juillet 1534 pour la levée de sept légions d'infanterie ${ }^{8}$. Peu de temps après, ce droit est étendu à l'ensemble des soldats par l'ordonnance de novembre 1549 sur la levée, l'entretien et la police des gens de guerre ${ }^{9}$. Enfin, l'ordonnance de Blois de 1550 prévoit de consigner sur la solde des soldats une somme pour servir à l'établissement d'un " hospital ambulatoire ${ }^{10} »$. C'est le premier acte normatif mentionnant ce type de secours ${ }^{11}$. L'hôpital est situé non loin des combats.

À partir du XVII ${ }^{\mathrm{e}}$ siècle, les conceptions stratégiques, les moyens matériels et humains évoluent considérablement. La puissance destructrice de l'armée par les armes à feu ${ }^{12}$ et le nombre de soldats engagés dans les combats augmentent. Dès la fin du XVI ${ }^{\mathrm{e}}$ siècle, et surtout au XVII ${ }^{\mathrm{e}}$ siècle, les ouvrages de chirurgien foisonnent. Les œuvres d'Ambroise Paré sur le sujet sont précoces ${ }^{13}$, elles montrent le savoir-faire des praticiens. Les traitements présentés permettent de saisir ce qu'endure le soldat sur le champ de bataille. La nouveauté des plaies par armes à feu intrigue ces professionnels à tel point que leurs traités développent des thérapies spécifiques ${ }^{14}$. À ces exigences chirurgicales nouvelles, s’ajoute la nécessité de soigner des affections purement médicales que la promiscuité des soldats favorise. En effet, les militaires sont touchés l'été et l'automne par des épidémies de malaria, appelée alors fièvre hélode ${ }^{15}$, de dysenterie bacillaire et de choléra-morbus ${ }^{16}$. Dès lors, la présence d’hommes de l'art se généralise dans les régiments. Ainsi, dans l'infanterie, un chirurgien fait

7 ISAMBERT, DECRUSY et JOURDAN, Recueil général des anciennes lois françaises..., t. 12, Paris, BelinLeprieur, 1828, p. 2 sq., art. 31 : «S'il advenoit qu'aucuns desdicts gens de guerre se transportassent malades en leurs maisons, ou ailleurs, parquoy ils ne peussent pas eux trouver à la monstre : le roy veut et ordonne que le capitaine, ou quatre des plus gens de bien de la compagnie certifieront que celuy qui sera ainsi malade, est homme de bien, et qu'ils ont esté deuëment certifiez et acertenez de ladite maladie, et en ce cas, ledit commissaire le mettra au roolle de ladite monstre comme present : toutefois, le roy n'entend pas que les excusations se facent, sinon pour une monstre seulement ».

8 Ibid., p. 390 sq., art. 39 : «Si un compagnon a esté blessé des ennemis, ou semblablement s’il est malade, en ce cas il sera payé de sa solde, et défend le roy qu’on y face aucun abus ou tromperie, sur peine d'en estre puni, sans aucune grace : et seront iceux blessez et malades par les commissaires et controlleurs, qui feront les monstres ".

$9 \quad$ Ibid., t. 13, p. 119 et suiv., art. 25 et 28.

10 «A toutes les monstres qui se feront, il sera prins sur la paye de chacun soldat un sol par écu pour estre convertis tant en magasin d'armes qu'à un hospital ambulatoire ", cité par Jean IMBERT, Le droit hospitalier de l'Ancien Régime, Paris, PUF, 1993, p. 45.

11 Albert-Charles Poncin, «Le Règne de Louis XI et l'organisation du service de santé militaire ", thèse de médecine, Université de Lyon, 1944, p. 19 ; également DES CILlEULS, «Le Service de santé militaire... ", art. cit., et IMBERT, ibid.

12 John-Forrest HAYWARD, Les armes à feu anciennes, vol. 1 : 1500-1660, vol. 2 : 1660-1830, Fribourg, Office du livre, 1963 ; Jacques DE GHEYN, Maniement d'armes, d'arquebuses, mousquets, et piques, en conformité de l'ordre de Monseigneur le prince Maurice, prince d'Orange, Amsterdam, R. de Baudous et H. Laurens, 1608.

13 Ambroise PARÉ, La méthode de traicter les playes faictes par haquebutes et aultres bastons à feu ; et de celles qui sont faictes par fleches, dardz et semblables ; aussi des combustions spécialement faictes par la pouldre à canon, Paris, V. Gaulterot, 1545 ; et id., Les œuvres d'Ambroise Paré, Conseiller et premier chirurgien du Roy. Divisées en vingt-neuf livres, avec les figures et portaits, tant de l'anatomie, sue des instruments de chirurgie, et plusieurs montres. Revuës et augmentées par l'autheur peu avant son décès, Paris, Veuve Gabriel Buon, 1598.

14 Scipion ABEILle, Le parfait chirurgien d'armée, le traité des playes d'arquebusade, le chapitre singulier tiré du guidon, l'anatomie de la teste et de ses parties, pour l'instruction des Etudians en chirurgie, Paris, J. Guignard, 1696 ; Julien LE PAULMIER, Traicté de la nature et curation des playes de pistolle, harquebouse, et autres bastons à feu, ensemble les remèdes des combustions et bruslures externes et superficielles, Caen, chez Pierre Philippe, 1569 ; A. BELloste, Le chirurgien d'hôpital, enseignant une manière douce et facile de guérir promptement toutes sortes de playes, Paris, chez Laurent d’Houry, 1696. 
partie de l'état-major pour le soin du corps. Des appointements lui sont versés ${ }^{17}$. À côté du chirurgien, la présence d'un aumônier ${ }^{18}$ est attestée pour le soin des âmes.

Néanmoins, le traitement des soldats blessés s'avère peu efficace en pratique ; les limites de ce système sont apparues lors de la Guerre de Trente Ans ${ }^{19}$. De fait, lorsque le rétablissement des soldats demande plus que des pansements, les pratiques antérieures de secours resurgissent : les malades et les blessés sont évacués à l'arrière, dans des hôpitaux civils ${ }^{20}$, où des lits leur sont réservés. Le roi dédommage la réquisition de lits sur la base d'un prix de journée par soldat ; les administrateurs n’y répugnent pas, même lorsque les fondateurs de l'institution n'avaient pas prévu le soin des soldats.

L'armée devient un terrain d'expérimentation administrative ${ }^{21}$. L'hôpital militaire, en particulier, offre un cadre propice à l'application d'idées nouvelles ${ }^{22}$. Militaires et historiens se sont ainsi beaucoup attardés sur l'ordonnance du $1^{\mathrm{er}}$ janvier 1747 , préparée par le secrétaire d'État à la guerre d'Argenson ${ }^{23}$. Elle est capitale pour le droit hospitalier militaire de la fin de l'Ancien Régime: toutes les ordonnances qui lui succèdent s'y réfèrent. Cependant, son importance ne fait pas d'elle une ordonnance constitutive car, comme l'indique son préambule, elle « rassemble en un seul corps les dispositions des ordonnances et règlements concernant les hôpitaux militaires $»^{24}$. Dès lors, il ne peut être soutenu que le droit hospitalier militaire naît en 1747.

Nous pouvons d'ores et déjà avancer qu'avant cette date il existe un droit applicable aux hôpitaux militaires ${ }^{25}$; essentiellement issu de la pratique, il se transcrit dans des textes

15 La fièvre hélode se contracte dans les marécages, v. É. Littré, Dictionnaire de la langue française, t. 2, Paris, L. Hachette, 1873-1874, p. 2002, col. 1, verbo « Hélode ».

16 Monique LUCENET, «Les épidémies dans l'infanterie au cours de la première moitié du XVIII ${ }^{\mathrm{e}}$ siècle », Forces armées et sociétés, Centre d’histoire militaire et d'études de défense nationale, 1987, p. 209212.

17 Voir l’ordonnance de janvier 1629, dite Code Michau, dans IsAMBERT, Recueil général..., op. cit., t. 16, p. 225-344.

18 La présence des aumôniers dans l'armée est plus ancienne, il en existe des traces dès l'époque franque, ils étaient appelés abbas in castris, voir Michel BRUNETEAU, «L'aumônerie catholique des forces armées. Normes canoniques, droits français et comparaisons européennes », thèse d'histoire du droit, Paris XI, 1999, p. 12.

19 Bernhard KROENER, « Conditions de vie et origine sociale du personnel militaire subalterne au cours de la guerre de trente ans », Francia, t. 15, 1987, p. 336.

20 Henri Leclair relate une telle prise en charge à l'hospice Comtesse de Lille en 1342, lorsque Philippe VI de Valois porta secours à la cité de Tournai, assiégée par Édouard III : Henri LECLAIR, «Les Hôpitaux militaires de Lille avant la Révolution. Essai historique », Société d'études de la province de Cambrai, recueil 17, 1925, p. 17. D’autres auteurs ont confondu ces établissements avec les hôtelsDieu et même les couvents : cf. Marie-Raoul BRICE et Maurice BOTTET, Le corps de santé militaire en France, son évolution, ses campagnes, 1708-1882, Paris, Berger-Levrault, 1907, p. IX.

21 Sur l'établissement des institutions militaires sous Louis XIV, voir André CORVISIER, Histoire militaire de la France, Philippe Contamine (dir.), t. 1, Des origines à 1715, Paris, PUF, 1997, p. 389, pour qui « les institutions militaires de la France ont sans cesse évolué de manière à la fois raisonnée et pragmatique ».

22 Sur ces aspects de la gouvernance des hôpitaux militaires, on se reportera également à Renaud LIMELETTE, «La gouvernance du service de santé des hôpitaux militaires de la réforme de 1747 à 1789 », dans Gouvernance, Justice et Santé, CHJ Éditeur, 2020, p. 145-174.

23 On lira avantageusement le bel ouvrage d'Yves COMBEAU, Le comte d'Argenson. Ministre de Louis XV, Paris, École des Chartes, 1999.

24 Pierre de BRIQUET, Code militaire, ou compilation des ordonnances des rois de France, concernant les gens de guerre, Paris, Brault père, 1761, p. 234.

25 Pourtant, à la fin du XVIII ${ }^{\mathrm{e}}$ siècle, la période antérieure à 1747 est décriée : « Avant l’ordonnance du $1^{\mathrm{er}}$ janvier 1747, qui fixe la forme de l'administration des hôpitaux, ce service, sans règles et sans principes permanens, étoit l'image du désordre et de la source de l'abus » (Des Hôpitaux militaires, Metz, Imprimerie de Collignon, 1789, p. 8). 
normatifs. Ce sont les usages des acteurs de l'hôpital militaire, du secrétaire d'État à la guerre au personnel de santé, qui façonnent le droit hospitalier militaire. La législation royale ne confirme la règle qu'une fois qu'elle semble bien établie et proportionnée au but de l'institution. Ce droit est construit a posteriori.

Après 1747, l'élaboration du droit hospitalier militaire change de forme. Les dispositions normatives s'assemblent pour former un système déductif ${ }^{26}$, réputé parfait dès sa conception. Que penser d'une telle réglementation ? La réforme rationnelle proposée en 1747 poursuit un but nouveau, qui s'éloigne de la finalité principale. Avant 1747, l'objectif était d'assurer des soins hospitaliers aux soldats malades ou blessés; dorénavant, le contrôle budgétaire de l'institution prend le dessus sur le soulagement des soldats. Cette nouvelle finalité a des conséquences sur le personnel de santé affecté dans les hôpitaux militaires. Une stricte hiérarchie et un contrôle des tâches sont imposés aux médecins, aux chirurgiens et aux apothicaires, et l'ensemble du personnel devient dépendant du pouvoir central.

Ainsi l'évolution du service de santé militaire sous l'Ancien Régime est marquée par une césure. S'il existait avant 1747 une certaine liberté provinciale, il n’en est plus de même après cette date : c'est l'uniformisation nationale qui s'étend sur tous les hôpitaux militaires.

\section{L'établissement du service de santé militaire}

C’est au début du XVII ${ }^{e}$ siècle que s'établit en France un service de santé militaire, quand apparaissent les premiers hôpitaux militaires. Pour les desservir, un personnel de santé doté d'un statut particulier y est affecté.

\section{L’organisation du service de santé}

Avec l'apparition des hôpitaux sédentaires et fixes, l'assistance aux militaires a pris un nouvel élan, car ces hôpitaux sont chargés d'accueillir les malades et les blessés en retrait des combats de façon durable ; l’hôpital sédentaire est aménagé de façon temporaire non loin des combats, alors que l'hôpital fixe est une construction durable et éloignée du champ de bataille. L'implantation et la répartition des hôpitaux sédentaires, de cinq lieues en cinq lieues à l'arrière des combats, reviennent au général commandant l'armée ${ }^{27}$. Le premier hôpital fixe en France ${ }^{28}$ est celui de Pignerol, créé par Richelieu en $1629^{29}$. Avec le secrétaire d’État à la guerre de Sublet de Noyers, ces hôpitaux sont établis dans des bâtiments préexistants, ou des plans sont tracés pour en construire ${ }^{30}$. Ainsi Dunkerque est doté d'un tel hôpital en 1649. Selon Richelieu, ces hôpitaux procurent l'avantage de remettre sur pied des soldats déjà aguerris au combat. Plus intéressante est la vision de Mazarin, qui ressent comme une priorité absolue le développement de l'institution, non seulement par charité, mais également par bienveillance $^{31}$ envers les soldats malades et blessés ${ }^{32}$. Les hôpitaux militaires fixes, sous l'impulsion de Louvois, se multiplient dès 1661. Les places exposées à l'ennemi, en particulier en Flandre et en Alsace, sont les premières dotées de tels hôpitaux. Vauban

26 Jean ChAgniot, « Les fins de guerres au XVIII ${ }^{\mathrm{e}}$ siècle en Europe », dans De la guerre à la paix, Paris, Economica, 2001, p. 80.

27 DES CilleUls, « Le Service de santé militaire... », art. cit., p. 23.

28 Le premier hôpital sédentaire en Europe est celui de Malines, établi en 1585, après la réquisition de la demeure d'un seigneur calviniste: Lucienne Van MEERBEECK, «Le service sanitaire de l'Armée espagnole des Pays-Bas à la fin du $\mathrm{XVI}^{\mathrm{e}}$ et au XVII ${ }^{\mathrm{e}}$ siècle ", Revue internationale d'Histoire militaire, 1959, n²0, p. 484.

29 D’après Antoine BALLAND, Les pharmaciens militaires français, Paris, L. Fournier, 1913, p. 1.

30 DES Cilleuls, « Le Service de santé militaire... », art. cit., p. 7.

31 Sur la distinction entre charité et bienveillance et leur rapprochement, on lira spécialement, Thomas d'AQuin, Somme Théologique, IIa, IIae, q 31, art. 1 sq.

32 Cité par Jean MEYer, Le poids de l'État, Paris, PUF, 1983, p. 92. 
complète le réseau hospitalier militaire en aménageant le pré carré : dans chaque place un hôpital militaire est fixé3 ${ }^{33}$ C'est à cette époque que sont construits les hôpitaux de Lille (appelé hôpital royal), d'Ypres, de Tournai et de Maubeuge. Au début du XVIII ${ }^{\mathrm{e}}$ siècle, l'édit de janvier 1708 sur la création des offices de médecin et chirurgien dénombre une cinquantaine d'hôpitaux militaires. Dans les provinces du Nord, outre ceux déjà mentionnés, un chapelet de villes sont pourvues d'un hôpital militaire fixe : Valenciennes, Furnes, Condé, Cambrai, Givet et Charlemont, Gravelines, Philippeville, Le Quesnoy, Landrecies, Avesnes, Calais, Bergues et Rocroi. S'y ajoutent les hôpitaux établis non loin de là, comme en Artois : Arras, Saint-Omer, Béthune, Hesdin et Aire.

Au commencement du XVIII ${ }^{\mathrm{e}}$ siècle, les secours apportés aux soldats, aussi bien en temps de guerre qu'en temps de paix, sont organisés : les militaires bénéficient d’hôpitaux d'armée pour les secourir au plus près des combats, d’hôpitaux sédentaires reculés du champ de bataille qui permettent d'évacuer les blessés et de poursuivre les secours, et d'hôpitaux fixes où les soldats sont soignés dans la durée en des lieux plus commodes à la pratique de la chirurgie et de la médecine.

Le point commun de tous ces hôpitaux est qu'ils ont pour but la médicalisation du soldat, qu'il soit malade ou blessé. En ce sens, ils sont plus spécialisés que toutes les formes d'hôpitaux civils ${ }^{34}$. C'est cet objectif particulier qui nous semble le plus approprié pour définir l'hôpital militaire. Ce dernier se distingue de l'hôpital civil, non seulement par le fait qu'il ne reçoit que des militaires, mais aussi par l'absence de personnalité morale : l'hôpital militaire est un service de l'État, sous la direction du secrétaire d’État à la Guerre. Sans autonomie financière et décisionnelle, les hôpitaux militaires sont administrés de façon uniforme. Pour preuve, les hôpitaux de charité qui reçoivent des militaires sont dits « sous le pied militaire $»^{35}$.

La majorité des hôpitaux militaires sont situés le long des frontières du royaume. Leur importance numérique, nous conduit à n'étudier que ceux présents dans les intendances de Flandre et du Hainaut.

Le cadre géographique de ces deux intendances est particulièrement justifié, car ces territoires sont les théâtres de guerres sous les règnes de Louis XIV et de Louis XV, ce qui conforte l'existence d'hôpitaux militaires. La Flandre et le Hainaut sont, à partir du règne de Louis XIV, des intendances frontières dont les villes fortifiées forment le pré carré de Vauban $^{36}$. L’implantation des hôpitaux militaires commence dès la création de ces intendances et s'étend sur tout le ressort de celles-ci.

Une carte des hôpitaux militaires fixes peut être dressée à partir des hôpitaux mentionnés dans l'édit de 1708. Joint à cet édit, un arrêt du Conseil royal des finances du 17 janvier 1708 permet une classification des hôpitaux selon l'importance que le roi a voulu leur donner à travers le montant des appointements alloués aux officiers de santé (cf. carte). Parmi les cinquante hôpitaux recensés dans l'édit, la plupart sont installés dans le ressort des

33 Camille Rousset, Histoire de Louvois et de son administration politique et militaire, t. 2, Paris, Didier, 1864, p. 127.

34 Françoise HiLdeSHEIMER et Christian Gut, L'assistance hospitalière en France, Paris, Publisud, 1992, p. 57.

35 AN, Z ${ }^{1 \mathrm{C}}$ 184, Extraordinaires des guerres, département du Hainaut, compte du mois de janvier 1726. Dans les comptes de l'extraordinaire des guerres, les hôpitaux de Maubeuge, d'Avesnes, de Landrecies, de Philippeville et de Givet sont dits royaux et celui de Valenciennes, administré par les dames religieuses de l'Hôtel-Dieu, est dit sous le pied militaire. AD Nord C 5475, Lettre du Maréchal de Ségur à l'intendant Sénac de Meilhan, au sujet du paiement des fournitures fait par avance dans les hôpitaux de charité sur le pied militaire, fo 2 : dans cette correspondance il est clairement mentionné qu'en Hainaut-Cambrésis les hôpitaux de Cambrai, du Quesnoy et de Valenciennes sont « de charité sous le pied militaire ».

36 Anne BlANCHARD, «Les ingénieurs du Roy de Louis XIV à Louis XVI : étude du corps des fortifications », Thèse de lettres, Montpellier, Université Paul Valéry, 1979. 
intendances de Flandre et de Hainaut. Sur les dix hôpitaux militaires les plus importants ${ }^{37}$, six appartiennent à ces deux intendances : Dunkerque, Ypres, Lille, Tournai, Valenciennes et Maubeuge. Le même constat peut être fait pour les hôpitaux de moindre importance ${ }^{38}$. Ainsi, près de la moitié des hôpitaux militaires fixes du royaume sont situés en Flandre et en Hainaut.

\section{Carte : L'implantation des hôpitaux militaires d'après l'édit de janvier $1^{3708}{ }^{39}$}

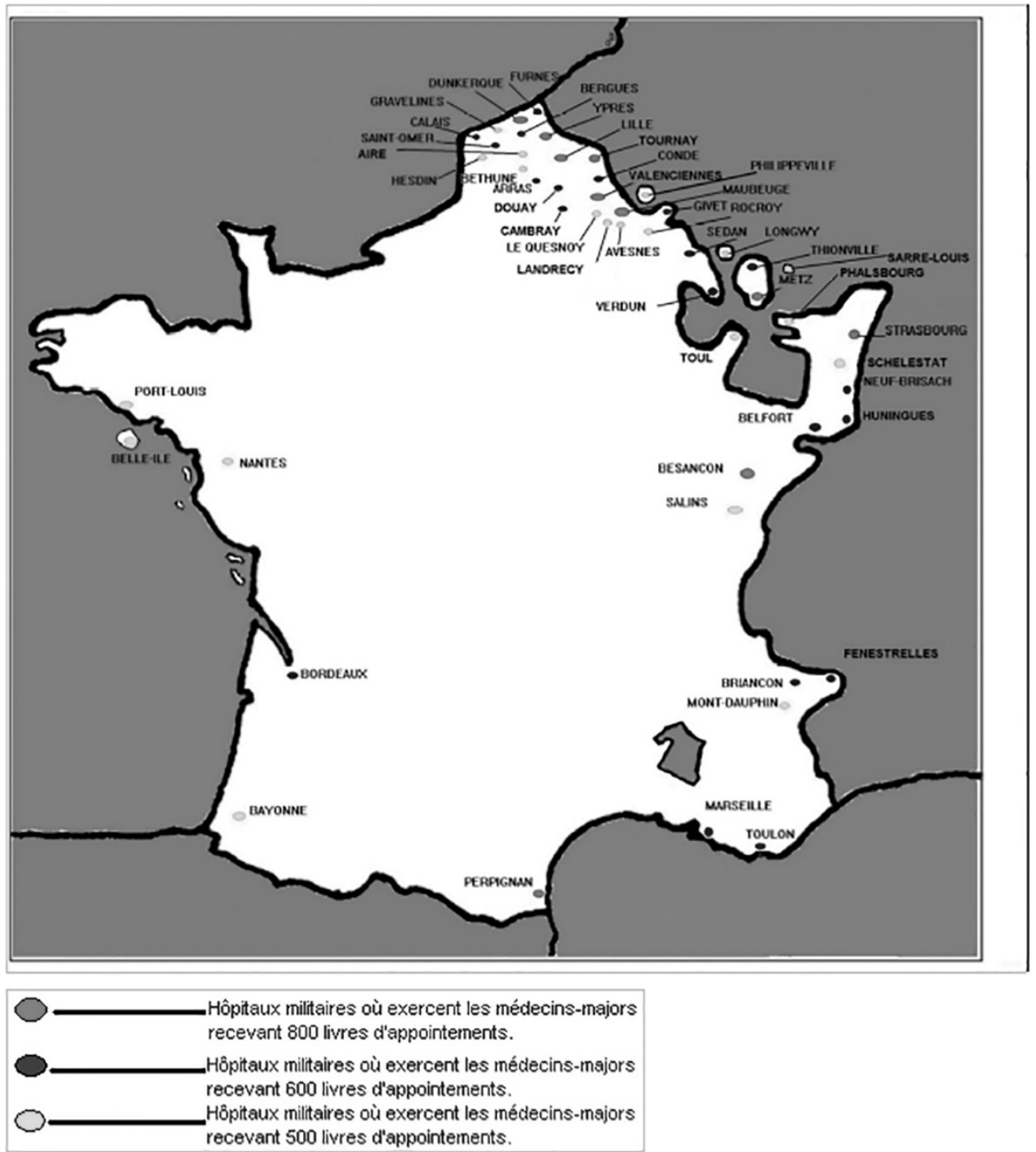

Les guerres des dernières décennies du XVII ${ }^{\mathrm{e}}$ siècle et du début du XVIII ${ }^{\mathrm{e}}$ siècle ${ }^{40}$ justifient également l'établissement d'hôpitaux ambulants et sédentaires, qui traitent les soldats des armées, en plus des hôpitaux fixes chargés plus particulièrement des troupes en garnison. Leur choix d'implantation est lié aux circonstances. L'établissement des hôpitaux ambulants et des hôpitaux sédentaires dépend par nature de la position des armées, celui des hôpitaux militaires fixes des disponibilités immobilières des villes et des places des intendances. C'est pourquoi l'implantation des hôpitaux militaires est le fruit du travail, soit de l'intendant, soit du commissaire des guerres, qui sont de par leurs fonctions sur le terrain les mieux placés

37 Ils sont recensés par une pastille rouge sur la carte.

38 Ils sont signalés en bleu et en vert sur la carte.

39 Nous avons conservé l'orthographe de l'édit de janvier 1708.

40 Rappelons qu'entre 1667 et 1714 trois guerres se sont déroulées sur le territoire des provinces du Nord : la guerre de Dévolution, la guerre de la Ligue d'Augsbourg et enfin la guerre de Succession d’Espagne. 
pour opérer les choix. Pourtant, à lire la bibliographie sur le sujet, tout semble être conçu à Paris ou à Versailles : cette vision centralisatrice, en faisant la part belle au premier ministre avant le règne personnel de Louis XIV, puis au roi et au secrétaire d'État ayant le département de la Guerre, occulte le travail en amont et fait de l'intendant et du commissaire des guerres de simples exécutants ${ }^{41}$.

Pourtant, les sources attestent du rôle particulier de l'intendant dans l'établissement des hôpitaux militaires. La commission particulière qu'il reçoit écarte tout doute sur ses attributions. En effet, il est compétent pour l'établissement des hôpitaux « tant à la suitte [sic] et près de nos armés ", c'est-à-dire dans l'ordre les hôpitaux ambulants et les hôpitaux sédentaires, "que [ceux] des garnisons [et] places ", c'est-à-dire les hôpitaux militaires fixes $^{42}$. Pour remplir cette fonction l'intendant est choisi en raison de sa «capacité, prévoyance Vigillance [sic] et affection »; ces termes confirment le rôle de conseiller de l'intendant : il est l'homme du roi.

Le commissaire des guerres intervient aussi dans les décisions concernant les hôpitaux militaires. Chargé de la police militaire, il doit prévoir, lors de la revue des troupes appelée « montre ${ }^{43}$, toutes les difficultés des étapes ${ }^{44}$. C'est à ce titre qu'il concourt avec l'intendant à la gestion des hôpitaux militaires. Ainsi à Utrecht, en 1672 durant la Guerre de Hollande, le commissaire des guerres Heiss s'oppose à la réunion des trois hôpitaux d'armée ${ }^{45}$. De même à Saint-Venant en 1675, le commissaire des guerres Benoist rend compte dès son arrivée des nécessités de l'hôpital. Il présente un mémoire sur l'administration de l'hôpital, qui malheureusement fait défaut dans les papiers conservés ${ }^{46}$. La perte du mémoire nous empêche de connaître les réformes proposées par Benoist, mais sa correspondance avec Le Tellier, alors secrétaire d'État ayant le département de la Guerre, confirme le rôle de conseiller des commissaires des guerres au sujet des hôpitaux militaires.

À Lille, l'action de l'intendant, aidé des commissaires des guerres, se mesure peu après le rattachement de la ville à la France ${ }^{47}$. En effet, le 7 octobre 1667, une épidémie de peste se propage dans la ville. Les militaires atteints sont isolés dans une dépendance de l’Hospice

41 Ainsi, Poncin écrit que c’est Mazarin qui fait établir l'hôpital ambulant à Mardyck et l'hôpital sédentaire à Calais, lors de la campagne de Flandre de 1658. L'intendant d'armée Blondot est seulement chargé d'organiser et de surveiller la fourniture de ces hôpitaux : voir Albert-Charles PonCIN, Le Règne de Louis XIV et l'organisation du service de santé militaire, Lyon, Imprimerie Paquet, 1944, p. 33-38.

42 Service Historique de la défense (SHD), Ya 1, « Traité du Formule du secrétaire d'estat à la guerre sous les regnes des Roÿs Henry IV surnommé Le Grand. Louis XIII. Et commencement de Louis XIV, Commission d'Intendant des hospitaux en Barrois, Lorraine, Alsace, et Allemagne », $\mathrm{f}^{\circ} \mathrm{58}, \mathrm{v}^{\circ}$ et $\mathrm{f}^{\circ} \mathrm{59}$, $r^{\circ}$. Notons que cette commission est retranscrite dans un formulaire du secrétariat d'État à la Guerre, elle servait donc d'exemple de commission d'intendant des hôpitaux et était usitée en Flandre et en Hainaut.

43 Georges GuILlET, Les arts de l'homme d'épée ou le dictionnaire du gentilhomme, Paris, chez la veuve Gervais Clouzier, $1682,2^{\mathrm{e}}$ partie, p. 187 et p. 188.

44 Selon Guillet, «c'est un officier étably [sic] pour la police militaire, et qui dans la marche des troupes, à l'œil sur les difficultés qui arrivent, pour la fourniture des étapes, et des ustanciles [sic], règle les billets des logements avec les habitants, et qui assiste aux montres et aux revües des gens de guerre », ibid., p. 79 et p. 80.

45 SHD, $\mathrm{A}^{1}$ 296, Guerre de Hollande, novembre et décembre 1672, doc. 47, lettre du commissaire des guerres Heiss du huit novembre 1672, il avance qu'un grand nombre de soldats blessés et malades subsiste lors des revues des régiments.

46 SHD, $\mathrm{A}^{14} 57$, doc. 35, Lettre de Benoist à Le Tellier du 29 novembre 1675.

47 Sur les conditions du rattachement de Lille à la France le 28 août 1667, voir « Lettres-Patentes du roi, pour l'enregistrement et l'exécution de la Capitulation accordée à la Ville de Lille, aux États et à la Chambre des Comptes », dans SIX et Plouvain, Recueil des Edits, Déclarations, Lettres-Patentes, etc., Douai, 1785, t. 1, p. 26-45. 
Comtesse $^{48}$, dans la maison dite du Prévost, faubourg de la Barre. Les soins apportés aux soldats sont financés par l'intendance ${ }^{49}$. Ce premier hôpital militaire lillois n'a cependant qu'une vocation temporaire. C'est en 1671 que le premier hôpital militaire fixe est créé pour soulager l'Hospice Comtesse ${ }^{50}$. Situé au-dessus de la porte Saint-Maurice, il fonctionne jusqu'en 1677. Enfin, en 1673, l'hôpital militaire Saint-Louis, aussi nommé hôpital royal, est établi non loin de la porte des malades, dans des casernes et écuries empruntées à la ville, cour du grand Flamend. Cet hôpital est conçu dès le départ pour accueillir les soldats en garnison $^{51}$. La situation juridique de l'hôpital militaire de Lille est spécifique. En effet, l'hôpital royal Saint-Louis, s'il ne reçoit que des militaires, n'est pas la propriété du roi : il appartient juridiquement à la ville ${ }^{52}$. Cette particularité permet de constater le travail de l'intendant et des commissaires des guerres dans la recherche d'un compromis entre le roi et la cité flamande.

En 1684, le roi veut faire réparer l'hôpital militaire par la ville car les casernes et les écuries affectées à l'hôpital royal Saint-Louis sont jugées vétustes, malsaines et incommodes pour la dispensation des $\operatorname{soins}^{53}$. Suite à l'évaluation du coût des travaux, jugé important, le commissaire des guerres Camus propose d'augmenter l'hôpital Saint-Louis sur un fond appartenant aux canonniers ${ }^{54}$. Comme la solution n'est pas retenue, l'intendant du Gué de Bagnols présente une alternative : rebâtir à neuf l'hôpital militaire. En définitive, devant l'opposition de la ville et après agrément de l'intendant, seuls des travaux d'agrandissement sont prévus ${ }^{55}$. Plus tard, de nouveau, l'intendant constate que l'hôpital est mal agencé ; entre 1689 et 1697, des travaux sont ainsi entrepris pour aménager une cour afin d'aérer l'édifice. Pour ce faire, la ville est contrainte, soit de racheter, soit de louer des fonds

48 Cet hospice ne recevait que des militaires depuis 1640 (LECLAIR, «Les hôpitaux militaires de Lille... ", art. cit., p. 24). Depuis la domination française, le roi dédommageait les religieuses en les exemptant, par grâces spéciales, de « toutes impositions royales et municipales mises et à mettre sur le vin qui a été et qui sera consommé audit Hôpital, jusques et à concurrence desdites quinze queues de vin par chacun an, de six autres d'augmentation, faisant en tout vingt-une queues ", dans Recueil des Edits, Déclarations, Lettres-Patentes..., op. cit., t. 1, p. 24-26, et PonCIN, Le Règne de Louis XIV et l'organisation..., op. cit., p. 59.

49 LECLAIR, « Les hôpitaux militaires de Lille... », art. cit., p. 28 et p. 29.

50 Pourtant des militaires sont encore envoyés à l’Hospice Comtesse jusqu’en 1751, voir « Mémoire de la ville de Lille concernant la construction d'un nouvel hôpital militaire Saint Louis », du 10 décembre 1751, f $\mathrm{f}^{\circ} \mathrm{r}^{\circ}$, Archives municipales de Lille (AM Lille), Affaires Générales (AG), cart. 302, dos. 22, et LECLAIR, « Les hôpitaux militaires de Lille... », art. cit., p. 32 ; sur les rapport entre les hôpitaux civils et les hôpitaux militaires et les inconvénients sur les pauvres malades de la cité, voir J. DES CILLEULS, «Le service de santé à l'intérieur sous l'Ancien Régime », Revue Historique de l'armée, n 3, août 1955, p. 54.

51 «Mémoire de la ville de Lille... », fo 1, v, AM Lille, A. G., cart. 302, dos. 22.

52 Ce n'est pas le cas dans les autres grands hôpitaux militaires du royaume, ceux-ci sont aménagés dans des immeubles abandonnés par des congrégations ou dans des bâtiments désaffectés appartenant au roi : $c f$. DES CILLEULS, « Le service de santé... », art. cit., p. 52.

53 Lettre de Louvois au maréchal d'Humières, gouverneur de la ville, du 23 juin 1684, AM Lille, A. G., cart. 302, dos. 22.

54 Extrait du registre du magistrat du 20 juillet 1684, dans « Mémoire de la ville de Lille... ", f $\mathrm{f}^{\circ}$, $\mathrm{v}^{\circ}$, et $\mathrm{f}^{\circ}$ 2, $\mathrm{r}^{\circ}$, AM Lille., A. G., cart. 302, dos. 22.

55 Extrait des registres du magistrat des 19 août 1684 et 2 juin 1685, dans « Mémoire de la ville de Lille... ", f ${ }^{\circ} 2$, $\mathrm{r}^{\circ}$ et $\mathrm{v}^{\circ}$, AM Lille, A. G., cart. 302, dos. 22. 
appartenant à des particuliers ${ }^{56}$, le tout après estimation, d'une part de la valeur du fonds ${ }^{57}$ et des édifices construits, d'autre part de la valeur de rendement ${ }^{58}$ du bien immobilier.

Il ressort des dossiers conservés aux archives municipales de Lille que l'intendant et les commissaires des guerres sont les mieux placés pour conseiller le roi lors de l'établissement de l'hôpital militaire Saint-Louis. Leur travail a permis de trouver un lieu pour implanter l'hôpital militaire ; leur présence dans la ville, lors des premières années de mise en service de l'hôpital, les a conduits à proposer un nouvel agencement de l'édifice pour le bien du service.

Dans d'autres villes moins importantes, les intendants conseillent Louis XIV et le secrétaire d'État ayant le département de la Guerre lors de l'établissement des hôpitaux destinés aux militaires. Pour préparer le siège de Mons au début de l'année $1691^{59}$, l'intendant d'armée du Gué de Bagnols a fait apporter dans l'hôpital militaire de Tournai des meubles et un mémoire des remèdes. Le roi confirme son initiative et l'invite à poursuivre les préparatifs ${ }^{60}$. Au début de la Guerre de Succession d'Espagne en février 1701, un détachement de troupes des garnisons de Dunkerque et de Furnes est envoyé pour entrer dans Nieuport et Ostende. L'intendant Barentin fait remarquer à Chamillart ${ }^{61}$, alors secrétaire d'État ayant le département de la Guerre, l'absence d'hôpital militaire dans ces deux villes. Il propose de ne rien faire pour Nieuport, car il est possible d'évacuer les malades vers Furnes ; pour Ostende il confie au commissaire des guerres Segent le soin de chercher un hôpital dans la ville pour un prix raisonnable. À défaut, il propose d'envoyer un ou deux frères bonfieux dans une maison particulière pour soigner les malades, en attendant la décision du roi ${ }^{62}$. Chamillart retient la proposition d'envoyer des frères bonfieux ${ }^{63}$. En avril, l'intendant suggère d'établir un hôpital militaire à Bruges selon le modèle suivi en Flandre maritime, en y plaçant un médecin, un chirurgien et un contrôleur. Pour des raisons de cherté des vivres et des ustensiles en Flandre espagnole, la fourniture sera assurée par l'envoi des frères bonfieux ${ }^{64}$. Cependant, quelques jours plus tard, l'intendant reçoit une offre d'un entrepreneur local ; Barentin la repousse aux motifs qu' " il est à craindre que les malades ne soient pas aussi bien servis par un entrepreneur qui n'a en vue que son profit, que par des religieux qui se sont consacrés au service des malades $»^{65}$. Outre les raisons financières, Barentin motive son choix par l'efficacité du service aux malades des frères bonfieux. L'hôpital accueillera les soldats malades de la garnison et ceux de l'armée en campagne.

Ici comme ailleurs, les intendants inspirent par leurs propositions la politique d'implantation des hôpitaux militaires ; le secrétaire d'État ayant le département de la Guerre, d'ailleurs, les y encourage. Ce dernier confie ainsi à l'intendant Bernières la tâche de suppléer aux

56 La procédure est menée par le procureur syndic de la ville Herreng, qui agit pour et au nom de la ville après autorisation du magistrat. Voir notamment l' « Autorisation des magistrats de la ville au procureur syndic du 6 mars 1694 », AM Lille, A. G., cart. 300, dos. 4.

57 L'estimation de la valeur du fonds est appelée prise ; voir notamment la « Prise d’une maison par la ville de Lille au-devant de l'hôpital de St Louis appartenant à François Willot », le 6 octobre 1689, AM Lille, A G, cart. 300, dos. 4, et l' « Achat d'une maison par la ville de Lille proche de l'hôpital de St Louis à François Willot », le 29 mai 1692, AM Lille, A. G., cart. 300, dos. 4.

58 Le rendement d'une maison est appelé rendage : Contrat de louage de deux petites maisons, entre le procureur syndic de la ville et François Willot, le 13 mai 1694, AM LILLE, A. G., cart. 300, dos. 4.

59 Roger RAPAILle, Le siège de Mons par Louis XIV en 1691 : étude du siège d'une ville des Pays-Bas pendant la guerre de la Ligue d’Augsbourg, Mons, Éditions du renard découvert, 1992.

60 SHD, A ${ }^{11}$ 043, doc. 56, Instruction du roi à M. du Gué de Bagnols, le 31 janvier 1691.

61 Emmanuel PÉNICAUT, Michel Chamillart: ministre et secrétaire d'État de la guerre de Louis XIV, Paris, École des chartes, 2004.

62 SHD, A ${ }^{11} 499$, Lettre de Barentin à Chamillart du 9 février 1701.

63 Ibid., Réponse de Chamillart à Barentin du 13 février 1701.

64 Ibid., Lettre de Barentin à Chamillart du 27 avril 1701

65 Ibid., Lettre de Barentin à Chamillart du 7 mai 1701, et réponse positive de Chamillart du 11 mai. 
désordres des hôpitaux militaires espagnols par ses propres suggestions sanitaires ${ }^{66}$. Au Sud, Bernières conseille d'ouvrir des hôpitaux supplémentaires à Louvain et aux alentours pour accueillir huit à neuf cents malades ${ }^{67}$. Chamillart lui répond de ne rien faire sans connaître la position des armées ${ }^{68}$. Fort de l'exhortation de Chamillart, Bernières suggère de garder le petit hôpital militaire de Namur de cinquante places, tant que la guerre n'est pas déclarée. En cas de conflit, il propose de transformer l'hôpital Notre-Dame, où sont installés les pauvres de la ville, en hôpital militaire. Avisé, il recommande de présenter prudemment ce changement à la ville de Namur, car il juge être dans « un pays de répliques », où il convient d' « apprivoiser les peuples $»^{69}$.

Une fois les différents types d'hôpitaux militaires établis, un personnel de santé spécifique pourvoyait aux nécessités du service.

\section{La pluralité des statuts du personnel de santé}

Les différentes structures hospitalières sont desservies par un personnel hospitalier dont l'importance varie selon le type d'hôpital. Dans les hôpitaux ambulants, il est restreint : des chirurgiens, des apothicaires et des aumôniers s'occupent des soins du corps et de l'âme. Dans les hôpitaux sédentaires, situés non loin des combats, des médecins complètent ce personnel. Enfin, dans les hôpitaux fixes, plus en retrait, des infirmiers parachèvent le service hospitalier. Avant l'édit de janvier 1708, portant création d'offices de médecins et de chirurgiens, aucun texte normatif ne réglemente de façon générale le statut du personnel de santé. Ainsi la charge des apothicaires des camps est érigée en titre d'office, celle des médecins et des chirurgiens est une commission, enfin les apothicaires des hôpitaux sédentaires ou fixes et les infirmiers exerçant dans ces derniers sont de simples employés de l'entrepreneur. Un rappel de la différence entre office et commission paraît nécessaire, car la distinction des statuts éclaire les buts du pouvoir royal dans la distribution des charges publiques au personnel de santé. En d'autres termes, quels sont les motifs qui conduisent le pouvoir royal à distinguer différents statuts pour le personnel de santé ?

Pour différencier les charges publiques que sont l'office et la commission, reportons-nous aux œuvres des juristes de l'Ancien Régime. L’office, selon Loyseau, est « dignité avec fonction publique $»^{70}$, de sorte qu'en droit le roi délègue une part de la fonction publique par un titre appelé « lettres de provisions ». Ce qui distingue l'office des autres charges, précise Domat dans Le droit public, c'est qu'il est toujours pour la vie, alors que les autres charges sont pour un temps ${ }^{71}$. De plus, il est des offices vénaux et non vénaux. Ces derniers sont attribués en raison de la seule dignité de la personne, alors que les offices vénaux sont donnés

66 Ibid., Lettre de Chamillart à Bernières du 11 mai 1701.

67 Ibid., Lettre de Bernières à Chamillart du 2 mai 1701.

68 Ibid., Réponse de Chamillart à Bernières du 5 mai 1701.

69 Ibid., Lettre de Bernières à Chamillart du 14 mai 1701. Concernant les termes de « répliques » et d' « apprivoiser » à propos du Hainaut et de la Flandre, on se rapportera avantageusement aux articles suivants : Jacques LORGNIER, « La justice du roi soleil dans les anciens Pays-Bas, organisation de la justice dans le ressort du conseil souverain de Tournai », dans Olav MOORMAN VAN KAPPEN (dir.), Les juridictions supérieures, Nimègue, G. Noodt Instituut, 1994, p. 19-52 ; Jacques LORGNIER, « Cour souveraine et parlement de Tournai, pièces maîtresses de l'ordre judiciaire français dans les anciens Pays-Bas », dans Jacques POUMARÈDE et Jack THOMAs (dir.), Les parlements de province, pouvoirs, justice et société du XV $V^{e}$ au XVIII ${ }^{e}$, Toulouse, Framespa, 1996, p. 141-164 ; Renée MARTINAGE, «Pouvoir royal et justice au parlement de Tournai (1668-1709), ibid., p. 165-190 ; Véronique DEMARS-SION, « Le parlement de Flandres, protecteur ou fossoyeur des particularismes », ibid., p. 191214, et Renaud LiMELETTE, « À la recherche de son juge dans le ressort du parlement de Flandre », dans « La résolution des conflits entre gouvernants et gouvernés », Journée d’étude tenue à Bruxelles le 8 février 2008.

70 Charles Loyseau, Les ouvres de maistre Charles Loyseau, avocat en parlement. Contenant les cinq livres du droit des offices, Paris, Chez Guillaume de Luyne, 1660, L. I, ch. II, col. 2, § 98, p. 7. 
sans discernement ${ }^{72}$. La commission, contrairement à l'office, n'est pas perpétuelle : elle dure et cesse selon la volonté du roi, qui peut révoquer le titulaire quand bon lui semble ${ }^{73}$. Comme l'office non vénal, la commission est attribuée à une personne digne, car les charges, où la confiance est nécessaire, sont remplies par des personnes capables ${ }^{74}$. En résumé, la combinaison de trois critères distingue les charges publiques : la dignité, la durée et la vénalité. La commission est donnée pour un temps à des personnes dignes sans considération financière. L'office est toujours donné pour la vie, soit selon un critère financier et sans discernement, il est alors dit vénal, soit sous condition de dignité et sans apport financier, il est alors dit non vénal. Ces trois critères sont étroitement liés aux buts poursuivis par le pouvoir royal.

Ainsi, jusqu'à l'édit de 1708, il n'existe pas de statut uniforme pour le personnel de santé. La fonction et le lieu de son exercice conditionnent le statut. À partir de cette date, selon AlbertCharles Poncin, le pouvoir royal établit un véritable Service de Santé en créant un corps de médecins et de chirurgiens militaires ${ }^{75}$. Pourtant, à lire le préambule où sont exposés les motifs de l'édit de janvier 1708, rien ne confirme cette position : les expressions « Service de Santé » et « corps de médecins et chirurgiens » sont absentes du texte. Ainsi les raisons qui ont poussé le pouvoir royal à modifier le statut des médecins et des chirurgiens militaires, audelà de l'insertion de ces mesures dans une politique circonstancielle et fiscale de mise en office, restent à préciser. En fait, l'analyse des édits de création et de suppression des offices de médecins et de chirurgiens fait apparaître que le pouvoir royal a voulu assurer un emploi certain pour les nouveaux officiers et un service continu de la médecine et de la chirurgie et, ainsi, profiter d'un personnel expérimenté et capable.

Le préambule de l'édit de janvier 1708 est divisé en deux parties. Dans la première, le roi rappelle son engagement dans la conservation et le soulagement des soldats. Sans revenir sur les mesures prises au siècle précédent, il annonce l'établissement dans les armées et dans les hôpitaux de médecins et de chirurgiens en titre d'offices. Dans une seconde partie, le préambule présente les avantages de la création d'offices par rapport à la commission. Deux avantages sont mis en avant : le caractère certain de l'emploi des officiers et la continuité de leur service.

La certitude de l'emploi est assurée tant que le médecin ou le chirurgien exerce sa charge publique auprès d'un corps de troupe ou dans un hôpital, et aussi dans l'hypothèse où il quitte le service, volontairement ou non. En effet, le dispositif de l'édit précise que les médecins et

71 Jean DomAT, Les loix civiles dans leur ordre naturel ; Le droit public, et Legum delectus. t. 2, Le droit public, Paris, Chez Bauche, 1756, L. II., T. I., préambule, p. 146, col. 2 et p. 147, col. 1 : « C’est par ce titre des provisions, que les Offices sont distingués de diverses charges, qui engagent à des fonctions publiques, telles que sont, par exemple, ces Charges qu'on appelle municipales, celle des Juge et Consuls des Marchands, celles des Commis qui exercent des recettes, ou d'autres fonctions, qui ne sont toutes que pour un tems, au lieu que les Offices sont pour la vie ; ainsi le mot Charge est un nom général commun aux Offices, et à ces autres fonctions ».

72 Ibid., S I, § 14, p. 151, col. 1 : « Mais il y a cette différence entre les Charges vénales et celles qui ne le sont point, que pour celles-ci c'est le Roi qui appelle aux Charges ceux que bon lui semble, sans que rien précède leur titre que le choix qu'il fait pour les donner à ceux qu'il en juge dignes : et pour les Charges vénales, il en donne les provisions sans discernement de personnes [...]».

73 Ibid., § 32, p. 154, col. 1 : « Les Commissions ne sont ni perpétuelles, ni pour un certain tems précis et réglé ; mais sont pour un tems indéfini, et durent ou cessent selon la volonté de celui qui a donné la commission, et il peut la révoquer lorsque bon lui semble »; et HENRY, au mot " commission », dans Joseph-Nicolas GUYOT, Répertoire universel et raisonné de jurisprudence civile, criminelle, canonique et bénéficiale..., Paris, Chez Visse, 1784, t. 4, p. 158.

74 Antoine Furetière, Dictionnaire universel, t. 1, Paris, SNL-Le Robert, 1978, au mot « Digne ».

75 A.-C. Poncin, « Aperçu sur l'organisation du Service de Santé militaire à la fin du règne de Louis XIV », Revue du Corps de Santé Militaire, t. 1, 1945, p. 417-419, et id., Le Règne de Louis XIV..., op. cit. 
chirurgiens retirés du service peuvent exercer en s'établissant dans une ville quelconque du royaume, sans faire partie du corps ou de la communauté des médecins ou des chirurgiens du lieu, à l'exception de Paris, où l'intégration dans le corps ou la communauté reste une condition préalable ${ }^{76}$. Les officiers peuvent s'établir lorsque les troupes ne sont plus en campagne ou lorsque les hôpitaux dans lesquels ils exercent ne subsistent plus. Plus largement encore, ils peuvent se fixer dans toutes les villes du royaume, même s'ils se sont personnellement retirés du service ${ }^{77}$. Quant aux professionnels en service, ils peuvent exercer leur art au lieu de leur domicile, sans être reçus dans les communautés organisées. Déjà, une ordonnance de novembre 1677 permettait au seul chirurgien-major des hôpitaux d'exercer dans la ville où l'hôpital était situé ${ }^{78}$.

Cette liberté, qui empiète sur les privilèges des médecins et chirurgiens jurés, a fait naître des conflits avec les praticiens civils ${ }^{79}$. Ainsi, à Valenciennes, la communauté des chirurgiens s'est souvent tournée vers le Magistrat de la ville ou l'intendant du Hainaut pour faire appliquer son privilège. La première trace de ce type de conflit remonte avant l'ordonnance de novembre 1677. En l'espèce, un règlement enjoignait à tous les chirurgiens de faire connaître au Magistrat les noms des blessés qu'ils soignaient, ceci pour permettre d'arrêter plus facilement les auteurs des blessures. Or bon nombre de blessés, pour éviter des poursuites, allaient se faire soigner chez les chirurgiens militaires, lesquels contrevenaient au règlement en cachant les noms des blessés. Pour remédier à ce désordre, le Magistrat de Valenciennes édicta une remontrance (16 décembre 1671), qui rappelait la répartition des fonctions des chirurgiens : aux chirurgiens militaires le soin des soldats, aux chirurgiens jurés le soin des bourgeois et manants de la ville. La formulation employée est sans équivoque : les chirurgiens militaires sont considérés comme des "estrangers " et "non admis juridicquement ", les chirurgiens de la ville comme des "domestiques ${ }^{80}$. Les termes employés sont riches de sens ; le domestique est celui qui est sous la loi de la domus ${ }^{81}$, la maison, qui doit s'entendre ici comme la communauté des chirurgiens jurés. Ainsi les chirurgiens militaires se trouvent en dehors de cette communauté, ils sont des étrangers, car ils n'ont pas suivi la formation en suivant un apprentissage de deux ans, ils n'ont pas accompli de chef-d'œuvre et n'ont pas prêté serment, si bien qu'ils ne peuvent exercer ${ }^{82}$.

76 Les privilèges de la Faculté de médecine de Paris et du collège de Saint-Côme pour les chirurgiens sont respectés, DES CILLEULS, « Le Service de santé militaire... », art. cit., p. 55 et p. 56.

77 L'édit prévoit par « caducité ou autrement ».

78 L'ordonnance est mentionnée dans un procès entre la communauté des chirurgiens de Valenciennes et Jean Clavely. Il est rapporté dans les pièces de ce procès que «l'intention du Roy estoit que le seul chirurgien-major de ladite ville y puisse tenir bouticle ouvert sans estre subject à la maîtrise de ladite ville ». Ce procès est retracé dans Ernest BouTON, «La corporation des chirurgiens-barbieurs à Valenciennes (1592-1760)», Mémoires historiques sur l'arrondissement de Valenciennes, t. 1, Valenciennes, E. Prignet, 1865, p. 47.

79 Sur la spécialisation des professions, on se reportera à Marie GATTI, La querelle des barbiers, chirurgiens et médecins (XIII ${ }^{e}$-XVIII ${ }^{e}$ siècles), thèse de doctorat de chirurgie dentaire, Université de Lorraine, 2014.

80 « Il n’est aucunement raisonnable de préférer les estrangers aux domestiques, et que d'ailleurs lesdits estrangers sont bien souvent inexperts et gens non admis juridicquement », ibid., p. 41.

81 Sur la notion de domus on lira spécialement : Elisabeth MAgNOU-NorTIER (dir.), Aux sources de la gestion publique, t. 1, Enquête lexicographique sur fundus, villa, domus, mansus, Villeneuve d'Ascq, Presses universitaires de Lille, 1993.

82 L'article 12 de la charte de 1592 des chirurgiens et barbiers de Valenciennes est rédigé comme suit : «Que personne, de quelle qualité ou condition qu'il soit, ne poldra exercer l'art de chirurgie ny barbier et tondre en ladite ville et banlieue, n'est qu'il ait accomply les dictz deux années d'apprentissage et chef d'œuvre et payé droictz cy devant mentionnés, aussy faict le serment en tel cas accoustumé sur paine d'encourir par chacun contrevenant et pour chacune fois en amende douze livres tournois au proffict de ladite chapelle, pour la seconde fois au double, et la troisième fois estre puny et corrigé à l'arbitraige de justice » (BOUTON, « La corporation des chirurgiens-barbieurs à Valenciennes... », art. 
Un autre procès, postérieur à l'édit de suppression des offices de médecins et chirurgiens militaires, reconnaît au seul chirurgien-major de l'hôpital et aux seuls chirurgiens jurés l'exercice de la chirurgie dans la ville ${ }^{83}$. L'affaire démontre, par ailleurs, que les chirurgiensmajors, qu'ils exercent par commission ou par office, peuvent simultanément assurer leur art à l'intérieur de l'hôpital, pour les militaires, et en ville, pour les habitants de celle-ci.

La continuité du service se comprend à travers la carrière proposée aux médecins et aux chirurgiens. L'édit crée douze charges différentes. Les médecins se voient proposer quatre offices de conseillers médecins-inspecteurs-généraux et cinquante de conseillers médecinsmajors pour les hôpitaux des villes frontières et places de guerre. Les offices de chirurgien sont plus nombreux en genre. Il est créé quatre offices de conseillers chirurgiens-inspecteursgénéraux, quatre offices de chirurgiens-majors de camps et armées de terre, cinquante offices de chirurgiens-majors des hôpitaux des villes frontières et places de guerre ${ }^{84}$, quatre-vingthuit à la suite des anciens régiments d'infanterie, quatre à la suite des compagnies des gardes du corps, deux à la suite des compagnies de mousquetaires, un à la suite de la compagnie des grenadiers à cheval, un à la suite de la gendarmerie, quarante-huit à la suite des anciens régiments de cavalerie et quinze à la suite des anciens régiments de dragons. Médecins et chirurgiens peuvent faire carrière dans l'armée en achetant, selon leur disponibilité financière, une charge de valeur supérieure.

Il ressort de cette première période que la création des hôpitaux militaires et la constitution d'un personnel de santé spécifique ne sont pas l'œuvre du roi ou même du secrétaire d'État ayant le département de la Guerre. Jamais le roi ou son Secrétaire n'a imposé une réglementation avant que les intendants ou les commissaires des guerres n'aient proposé une solution pratique en considération des possibilités locales. En définitive, les quelques règles de droit édictées ne sont intervenues qu'a posteriori et elles furent toujours le fruit d'une longue pratique éprouvée sur le terrain. C'est pourquoi il existe très peu de textes normatifs sur les hôpitaux militaires, du début du XVII ${ }^{\mathrm{e}}$ siècle au milieu du XVIII ${ }^{\mathrm{e}}$ siècle.

Ensuite, la tendance s'inversant nettement, éclosent une foule de textes normatifs de toute espèce : de l'édit au règlement, en passant par l'ordonnance. Désormais, l'hôpital militaire et son personnel doivent être réformés selon des objectifs définis directement par le secrétaire d’État ayant le département de la Guerre.

\section{La réformation rationnelle du service de santé militaire}

Au milieu du XVIII ${ }^{\mathrm{e}}$ siècle, le secrétaire d’État ayant le département de la Guerre impose une réforme rationnelle du service de santé militaire. Il est dès lors inconcevable que des conceptions différentes subsistent entre les provinces. Le mot d'ordre est l'unification du service dans un système, défini une première fois par l'ordonnance de janvier 1747. Ainsi, l’unification et la rationalité s’imposent au statut du personnel de santé.

cit., p. 39.

83 Dans leur requête, les chirurgiens de la communauté de Valenciennes demandent à l’intendant du Hainaut Machault d'Arnouville que l'aide-major de l'hôpital et les garçons chirurgiens cessent d'exercer la chirurgie dans la ville, cette liberté n’étant consentie qu'au chirurgien major. Dans son ordonnance du 13 janvier 1744, l'intendant Machault d'Arnouville répond favorablement à la requête des chirurgiens jurés en faisant « deffense aux garçons chirurgiens de l'hôpital royal d'exercer la chirurgie dans la ville et dépendances de Valenciennes, jusqu’à ce qu'ils soient admis au nombre des maîtres chirurgiens dans la communauté établie en ladite ville », ibid., p. 66.

84 Sur les cinquante hôpitaux mentionnés dans l'édit vingt-trois se situent dans les ressorts des intendances de Flandre et de Hainaut : Dunkerque, Ypres, Lille, Tournai, Valenciennes, Maubeuge, Arras, Saint-Omer, Calais, Bergues, Furnes, Douai, Condé, Cambrai, Givet et Charlemont, Béthune, Hesdin, Aire, Gravelines, Philippeville, le Quesnoy, Landrecies, Avesnes. 


\section{L’ordonnance de 1747}

Pour mieux saisir l'ordonnance de janvier 1747, il convient de s'attarder sur son initiateur, le secrétaire d'État à la guerre de l'époque, le comte d'Argenson.

Marc-Pierre de Voyer de Paulmy, né en 1696, est nommé secrétaire d’État à la Guerre en janvier 1743 après le décès de son prédécesseur François Victor Le Tonnelier de Breteuil. Le comte d'Argenson a travaillé activement à sa charge jusqu'à son renvoi en février 1757 ; il décède en 1764. Il a mené de nombreuses réformes : des fortifications en 1743, des grenadiers royaux en 1744, de l'artillerie en 1755, du Dépôt de la guerre en 1756. L'une des plus marquantes est sans nul doute celle instruite entre 1746 et 1747, qui portait précisément sur les hôpitaux militaires. Avant sa nomination à ce poste prestigieux, le comte d'Argenson fut tour à tour avocat du roi au Châtelet en 1717, conseiller au parlement de Paris et maître des requêtes en 1719, lieutenant général de police les premiers mois de l'année 1720, puis entre 1722 et 1724, mais aussi intendant de Tours de 1720 à 1722, et enfin chancelier du duc d’Orléans en 1723.

D’Argenson côtoie alors les plus grands de l'époque, le roi Stanislas, la reine Marie Leszczyńska et le président Hénault. Il compte aussi parmi ses proches les philosophes Diderot et d'Alembert - l'Encyclopédie lui est d'ailleurs dédiée. Voltaire le remercie vivement dans sa préface du siècle de Louis XIV, n'hésitant pas à lui attribuer la paternité de l'ouvrage. Cette proximité avec les philosophes s'exprime dans la réforme des hôpitaux militaires. Il y déploie un esprit de déduction, d'ordonnancement, de hiérarchie, de contrôle... autant de concepts qui sont la marque des Lumières.

D'emblée, on est frappé par la forme de l'ordonnance. Elle est le fruit d'un esprit ordonné où chaque élément, chaque thème, est abordé dans une partie spécifique. Son titre, de plus, est singulier : " Ordonnance portant règlement général concernant les hôpitaux militaires ». Un « règlement général » : le ton est donné. Tout ce qui suit n'est que prescription obligatoire. Avant même le préambule, qui ordinairement marque le début des ordonnances, une table des matières des trente-trois titres qui composent l'ordonnance permet au lecteur d'en saisir l'esprit et la logique. Les premiers titres concernent la réception des soldats, leur visite, la fourniture des médications et de l'alimentation, la sortie des soldats, leur décès. Les titres formant le cœur de ce règlement s'intéressent ensuite à l'économie des hôpitaux militaires ; ils s'arrêtent sur la forme des états de dépenses, sur le rôle particulier des commissaires des guerres, sur celui des contrôleurs, sur les obligations de l'entrepreneur chargé de fournir et garnir les hôpitaux. Ce n'est que dans les derniers titres du règlement qu'est définie pour chaque personnel de l'hôpital sa fonction : l'aumônier, le médecin, le chirurgien-major et enfin les inspecteurs des hôpitaux. Au premier coup d'œil, se dégage une idée maîtresse : la réforme des hôpitaux militaires se veut claire, chaque facette de l'institution fait l'objet d'un point particulier.

Vient ensuite le préambule. Dans la tradition légistique de l'ancien droit, le roi y expose avec soin ses motivations. Le préambule est le lieu où sont mis en relief les désordres de l'institution ; le roi y expose les abus qui sont remontés jusqu'à lui et propose succinctement le plan de la réforme, qui est développé ensuite dans le dispositif. Ici, le préambule est minimaliste :

Le Roy voulant rassembler en un seul corps les dispositions des ordonnances et règlemens [sic] concernant les Hôpitaux militaires, et suppléer à ce qui a paru y manquer, Sa Majesté a ordonné et ordonne ce qui suit.

La motivation est courte : rassembler en un seul corps les dispositions normatives antérieures et suppléer à ce qui a manqué. Ainsi, le grand travail de d'Argenson se résumerait à une compilation, avec certes quelques ajouts ? Nous verrons qu'il n'en est rien, que cette 
ordonnance ne s'est pas contentée de rassembler ce qui existait auparavant, mais qu'elle a bouleversé les pratiques et a redéfini toute l'institution militaro-hospitalière, en particulier le statut des officiers de santé.

Suite au préambule, les titres s'enchaînent dans l'ordre présenté dans la table des matières, chaque titre étant découpé en articles. Mais ce qui est plus neuf encore, c'est qu'à la fin de l'ordonnance, après le dernier titre, une table alphabétique des matières est dressée. Soixantecinq mots-clés traversent ainsi toute l'institution. On y trouve quelques références aux aliments, aux chemises, aux fossoyeurs, mais les mots les plus renseignés sont ceux qui portent sur les officiers de santé, comme les chirurgiens-majors, les chirurgiens aides-majors, les chirurgiens de garde, les apothicaires et les médecins. À côté des officiers de santé, d'autres personnages semblent avoir une place importante dans l'hôpital militaire défini par le comte d'Argenson. En effet aux mots " commissaires des guerres », " controlleurs [sic] », « directeurs », les références aux titres et aux articles sont fort nombreuses.

L'esprit de déduction, de rationalité que l'on soupçonne déjà dans l'analyse de la forme de l'ordonnance est confirmé par le fond. En effet, sans même s'attarder aux dispositions concernant la fourniture des hôpitaux militaires, déléguée à un entrepreneur, celles qui concernent les officiers de santé dressent une chaîne de commandement.

Dans chaque hôpital militaire, l'ordonnance place les officiers de santé sous la sujétion du commissaire des guerres ${ }^{85}$. Ainsi, ce dernier est considéré comme le chef administratif des officiers de santé, placés sous sa main ${ }^{86}$. Pour parfaire ce contrôle, il est adjoint au commissaire des guerres un contrôleur qui le supplée en cas d'absence ${ }^{87}$.

Entre les officiers de santé, l'ordonnance dessine également une hiérarchie. Au sommet, le médecin donne ses ordres à l'apothicaire en chef et aux garçons apothicaires. Il peut à cet égard congédier les garçons apothicaires en cas d'incapacité ou de manque d'assiduité ; cependant, il ne saurait le faire sans le consentement du commissaire des guerres. Il peut également demander le renvoi de l'apothicaire en chef au commissaire des guerres et à l'intendant, mais en cas d'urgente nécessité il peut le relever seul de ses fonctions ${ }^{88}$. En l'absence de médecin, c'est le chirurgien-major qui remplit ses attributions ${ }^{89}$. Dans tous les cas, le chirurgien-major est le chef de la chirurgie dans l'hôpital, et à ce titre commande les chirurgiens et les garçons chirurgiens ${ }^{90}$. C’est le chirurgien-major qui admet dans les hôpitaux

85 Ordonnance du $1^{\mathrm{er}}$ janvier 1747, portant règlement général concernant les hôpitaux militaires, titre vingt-deuxième, article premier : «tous les officiers et employés de chaque hôpital, sans aucune exception seront aux ordres du Commissaire des guerres, auquel ils rendront compte de leur conduite, et seront tenus de représenter leurs registres toutes les fois qu’il le requerra, à peine de désobéissance ».

86 Ibid., article deuxième : "Le Commissaire des guerres tiendra la main à ce que lesdits officiers et employés exécutent ce qui leur est prescrit par les articles précédens du présent règlement, et par ceux qui vont suivre ».

87 Ibid., titre vingt-troisième, article premier : « Le Contrôleur établi dans chaque hôpital suppléera aux fonctions du Commissaire des guerres en son absence, à l'exception néanmoins des cas de juridiction et des peines à prononcer, qui seront réservez audit Commissaire des guerres, pour y pourvoir à son retour, sur le compte qui lui en sera rendu par le contrôleur ».

88 Ibid., titre vingt-sixième, article deuxième : " L'Apothicaire en chef et les garçons apothicaires seront aux ordres principalement du médecin : aucun garçon apothicaire ne sera admis dans l'hôpital, qu'il n’ait été auparavant bien examiné par lui, ledit médecin devant être le maître de congédier lesdits garçons, et de les changer s'ils manquent de capacité et d'assiduité à leurs devoirs ; ce qu'il ne pourra faire néanmoins sans le consentement du Commissaire des guerres. À l'égard de l'apothicaire en chef, ou Major des aide-majors et sous-aide-majors, il instruira le Commissaire des guerres et l'Intendant des raisons qu'il y auroit de les renvoyer, afin qu'il y soit pourvû, et pourra même les interdire de toutes fonctions en cas de nécessité urgente, et jusques à nouvel ordre ».

89 Ibid., article troisième : « Dans les hôpitaux où il n’y aura point de médecin, ou en son absence, tout ce qui lui est prescrit par le présent règlement sera exécuté par le chirurgien-major ».

90 Ibid., titre vingt-septième, article premier : "Le chirurgien-major est et sera le chef de tous les chirurgiens, aide-majors et garçons chirurgiens de l'hôpital, qui seront tenus de lui obéir comme à leur 
militaires les garçons chirurgiens et qui examine leurs instruments ${ }^{91}$. Il ne peut les renvoyer sans le consentement du commissaire des guerres.

L'ensemble des officiers de santé et le commissaire des guerres sont sous l'autorité directe de l'intendant et du secrétaire d'État ayant le département de la Guerre. Mais l'intendant, bien que représentant du roi dans la province, n’a plus le même rôle qu'auparavant. Il n'a gardé dans ses compétences que sa fonction judiciaire ; tout au plus est-il un rouage administratif supplémentaire entre le commissaire des guerres et le secrétaire d'État. Désormais, tous les officiers de santé qui desservent les hôpitaux militaires sont placés sous l'autorité du secrétaire d'État ayant le département de la Guerre. L'ordonnance provoque ainsi une véritable centralisation de l'institution, destinée à unifier l'administration hospitalière.

Après 1747, si d'autres ordonnances sont venues réglementer ${ }^{92}$ l'institution, elles n'ont jamais bouleversé l'économie générale de celle du $1^{\mathrm{er}}$ janvier 1747 . La meilleure preuve est l'ordonnance du 2 mai 1781, qui confirme l'idée de centralisation avancée en 1747. Son préambule est beaucoup plus long, mais il précise avec force la continuité entre les deux textes :

Le ROI s'étant fait représenter toutes les ordonnances concernant les Hôpitaux militaires, [...], SA MAJESTÉ a jugé à propos de rassembler, en un seul corps, toutes les dispositions, tant anciennes que nouvelles, qui ont été reconnues utiles, et de régler invariablement, en prenant pour base l'Ordonnance de 1747, toutes les parties de cette Administration, par une seule loi qui suppléant toutes celles précédemment rendues sur ce service, dispensât d'y avoir recours désormais ${ }^{93}$.

La similitude entre les deux ordonnances est frappante. Tout comme la première, la seconde comprend une table des titres (37); chaque titre concerne un pan particulier de l'administration des hôpitaux et se décompose en plusieurs articles numérotés. L'ordonnance comprend aussi une table alphabétique des matières, ici numérotée de I à VI. Cette table compte cent vingt-neuf mots matières, qui renvoient aux titres et articles concernés. À la suite de la table des matières, se trouvent dix modèles imprimés pour la gestion des hôpitaux militaires. Ainsi dès la seconde moitié du XVIII ${ }^{\mathrm{e}}$ siècle, le pouvoir central impose avec vigueur ses volontés sur l'administration des hôpitaux militaires.

Les sanctions disciplinaires forment un parfait exemple des modalités de l'uniformisation des officiers de santé. À la fin de l'année 1787, les officiers de santé de l’hôpital militaire de Landrecies déplorent la mort du soldat Monnet. Son décès est la suite des complications d'une gangrène que le soldat avait développée à la jambe. Les chefs militaires de son régiment ont alors porté plainte directement devant le secrétaire d’État ayant le département de la Guerre à l'encontre du chirurgien-major Martin. Le motif de la plainte est le suivant :

supérieur, en tout ce qui concerne son art et son service ».

91 Ibid., article deuxième : «Il ne sera admis dans l’hôpital pour le service des malades ou blessés, aucun garçon chirurgien qui n'ait été auparavant examiné par le chirurgien-major, qui visitera aussi leur instrumens ; ledit chirurgien devant être le maître de les congédier et changer, s’ils manquent de capacité et d'assiduité à leurs devoirs ; ce qu’il ne pourra faire néanmoins sans le consentement du Commissaire des guerres ».

92 Voir les ordonnances suivantes : Ordonnance du 4 août 1772, concernant les hôpitaux militaires ; Ordonnance du 27 février 1777 concernant les hôpitaux militaires ; Ordonnance du $1^{\text {er }}$ janvier 1780 en vingt-cinq articles sur les hôpitaux militaires et le service de santé ; Code du $1^{\text {er }}$ janvier 1780 d'administration des hôpitaux militaires et de charité au compte du roi ; Ordonnance du 2 mai 1781. Il faut attendre l'ordonnance du 20 juillet 1788, sur la constitution et l'administration générale des hôpitaux militaires, pour que soient démantelés les hôpitaux militaires fixes au profit d’hôpitaux régimentaires.

93 SHD, Ya 127, Ordonnance du 2 mai 1781. 
Martin a soigné seul le soldat Monnet sans consulter le médecin de l'hôpital Dollez ${ }^{94}$. L'intendant Sénac de Meilhan ${ }^{95}$ demande alors au commissaire des guerres Lépine de lui rendre compte des motifs de la mésintelligence entre le chirurgien-major et le médecin ${ }^{96}$. Le rapport du commissaire des guerres est fort instructif quant au contrôle exercé sur les officiers de santé et l'uniformité qui doit en découler. En effet, il confirme que le chirurgienmajor n'a pas l'estime de ses collègues et de ses subordonnés. Selon Dassier, chirurgien aidemajor, la mésentente avec le médecin tient au fait que le chirurgien-major à une « exigence de bienséance et de prévenance ${ }^{97}$. Ainsi, pour le chirurgien-major Martin, il subsiste un esprit de corps qui différencie chaque discipline médicale ; il y a les chirurgiens d'une part, et les médecins d'autre part, chaque corps étant indépendant.

À la réception du rapport du commissaire des guerres, l'intendant juge le cas du soldat Monnet chirurgical et reconnaît que le procès-verbal du traitement, signé et attesté par le chirurgien aide-major, les élèves et l'apothicaire, montre que la mort n’a pu être évitée malgré le respect des principes de l'art. Tout semble plaider en faveur du chirurgien-major ; d'ailleurs, l'intendant remarque que Martin n'a pas eu de mauvaises intentions, et souligne qu'il mérite quelque considération pour ses services. Pour autant, il ajoute à l'adresse du secrétaire d'État, que "les officiers de santé doivent agir en toutes occasions de concert ", puis conclut : « Je pense que le chirurgien doit être fortement réprimandé ». Il propose alors de signifier à Martin qu'à la première plainte qui sera portée contre lui et qui prouvera qu'il n’y a pas eu le plus parfait accord entre lui et le médecin, il sera révoqué. Dès lors, l'intendant justifie les sanctions demandées contre Martin, non pour une faute dans l'exercice de son art chirurgical, mais en raison de l'absence de concertation avec le médecin. La décision du secrétaire d’État adopte ce raisonnement ; selon lui, l'attitude du praticien est en désaccord avec les dispositions du titre VI de l'ordonnance du 2 mai 1781. La sanction est rude : au prochain écart, Martin sera destitué de son emploi.

Ainsi, l'impulsion donnée en 1747 s'est poursuivie jusqu'à la fin de l'Ancien Régime. La centralisation de l'autorité administrative s'est manifestée par le développement d'une chaîne de contrôle hiérarchique, au sommet de laquelle est placé le secrétaire d'État ayant le département de la Guerre. Petit à petit, les modalités de contrôle qu'il a définies ont dégagé un statut uniforme du personnel de santé.

\section{L’unification du statut du personnel de santé}

Pour parachever le contrôle hiérarchique des officiers de santé militaires, ceux-ci ont, outre leurs tâches médicales et chirurgicales, l'obligation de remplir des formulaires afin de comptabiliser les soldats entrant et sortant. Ces formulaires participent à la rationalisation du service de santé hospitalo-militaire à travers un contrôle budgétaire régulier. Le modèle de ces formulaires a été envoyé dans tous les hôpitaux militaires afin de faciliter le contrôle par une procédure uniforme.

Ainsi les chirurgiens militaires doivent remplir le formulaire suivant ${ }^{98}$ tous les mois.

94 AD Nord, C 17 877, Procès-verbal du Commissaire des guerres de l'hôpital militaire de Landrecies de Lépine du 21 décembre 1787.

95 Sur cet intendant, on lira avantageusement Stefano GENETTI, «Considérations sur Sénac de Meilhan moraliste », dans Cahiers de l’AIEF, 2007, n 59, p. 221-235.

96 Ibid., Lettre de Sénac de Meilhan du 16 décembre 1787.

97 Ibid., Procès-verbal du Commissaire des guerres de l'hôpital militaire de Landrecies du 21 décembre 1787.

98 Mss. Ars. 2261, "État des Soldats, cavaliers, Dragons, ou autres, Malades et Blessés... », dans « Recueil de tout ce qui a été fait à l'occasion du nouvel arrangement des hôpitaux depuis le $1^{\mathrm{er}}$ février 1746 jusqu'au $1^{\text {er }}$ janvier 1749 », p. 69-71. 


\begin{tabular}{|c|c|c|c|c|}
\hline \multicolumn{5}{|c|}{$\begin{array}{l}\text { Hôpital d' } \\
174 \_ \\
\text {État des Soldats, Cavaliers, Dragons, ou autres, } \\
\text { Malades et Blessés, qui ayant été traitez dans ledit } \\
\text { Hôpital, se trouvent hors d'état de servir par leurs blessures ou infirmités. } \\
\text { Sçavoir }\end{array}$} \\
\hline $\begin{array}{c}\text { Noms } \\
\text { des } \\
\text { Régimens }\end{array}$ & $\begin{array}{c}\text { Noms } \\
\text { des } \\
\text { Compagnies }\end{array}$ & $\begin{array}{c}\text { Noms et } \\
\text { qualités } \\
\text { des } \\
\text { Malades ou } \\
\text { blessés }\end{array}$ & $\begin{array}{c}\text { Lieux } \\
\text { de leur } \\
\text { Naissance } \\
\text { et tems de leur } \\
\text { Services }\end{array}$ & Observations \\
\hline \multicolumn{5}{|c|}{$\begin{array}{l}\text { Nous Chirurgien-Major de l'Hôpital royal et militaire d } \\
\text { Certifions l'Etat ci-dessus véritable. À } \\
\text { Jour du mois d' } \quad \text { mil sept cent quarante- }\end{array}$} \\
\hline
\end{tabular}

L'exactitude du formulaire dressé par le chirurgien-major est contrôlée par un bureau de vérification des hôpitaux militaires, service qui dépend du secrétaire d’État ayant le département de la Guerre. En effet, ce bureau centralise tous les formulaires envoyés par les chirurgiens-majors des différents hôpitaux. Il compare alors les mentions de chaque formulaire avec celles renseignées sur d'autres formulaires établis par les commissaires des guerres et les contrôleurs.

Par exemple, les commissaires des guerres et les contrôleurs sont chargés de rédiger les billets de sortie pour les soldats ${ }^{99}$ :

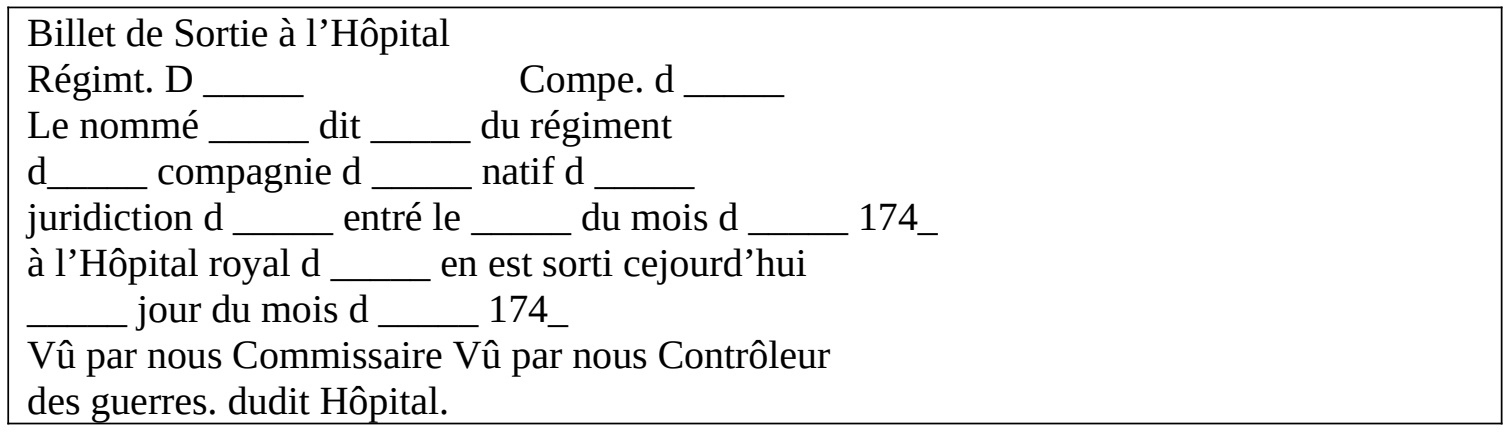

Ils dressent également un état des soldats qui restent dans l'hôpital, de ceux qui sont entrés malades ou blessés, de ceux qui en sont sortis, et de ceux qui y sont morts ${ }^{100}$.

99 Ibid., «Billet de sortie de l'hôpital », dans « Recueil de tout ce qui a été fait à l'occasion du nouvel arrangement des hôpitaux depuis le $1^{\text {er }}$ février 1746 jusqu’au $1^{\mathrm{er}}$ janvier 1749 », p. 73.

100 Ibid., « Vérifications de l’Etat des Soldats, Cavaliers ou Dragons qui restoient aud. hôpital, de ceux qui sont entrés malades ou blessés, de ceux qui y sont sortis, et de ceux qui y sont morts pendant ledit mois », dans « Recueil de tout ce qui a été fait à l'occasion du nouvel arrangement des hôpitaux depuis le $1^{\text {er }}$ février 1746 jusqu’au $1^{\text {er }}$ janvier 1749 », p. 109-112. 


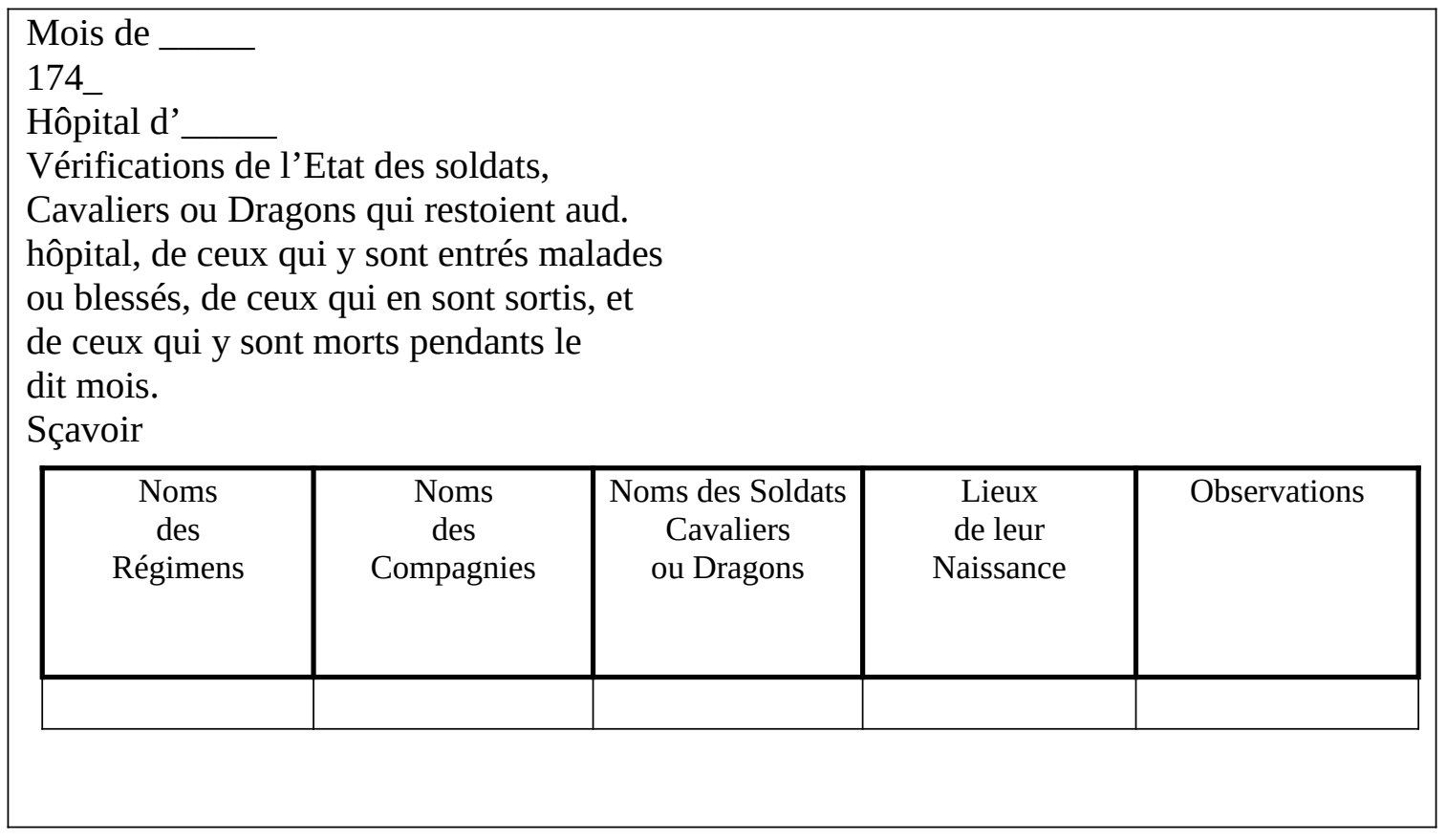

\begin{tabular}{|c|c|c|c|c|c|}
\hline \multicolumn{6}{|c|}{ Récapitulations } \\
\hline \multirow[b]{2}{*}{$\begin{array}{l}\text { Noms des } \\
\text { Régimens }\end{array}$} & \multirow{2}{*}{$\begin{array}{c}\text { Nombre et qualité } \\
\text { des Soldats, } \\
\text { cavaliers ou Dragons }\end{array}$} & \multirow[b]{2}{*}{$\begin{array}{c}\text { Nombres des } \\
\text { Journées }\end{array}$} & \multicolumn{3}{|c|}{ À déduire } \\
\hline & & & $\begin{array}{l}\text { Prix accordés } \\
\text { aux } \\
\text { entrepreneurs }\end{array}$ & $\begin{array}{l}\text { Retenue sur } \\
\text { les troupes }\end{array}$ & Total \\
\hline \multirow{3}{*}{\multicolumn{2}{|c|}{ 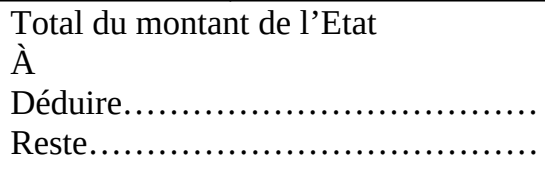 }} & & & & \\
\hline & & & & & \\
\hline & & & & & \\
\hline
\end{tabular}

Remarquons que ce dernier formulaire est quasiment identique à celui dressé par le chirurgien-major. Cette similitude permet ainsi en un seul coup d'œil de comparer rapidement les deux formulaires. Derrière ce contrôle budgétaire, apparemment anodin, le contrôle des officiers de santé se précise.

Lors de la préparation de l'ordonnance du $1^{\mathrm{er}}$ janvier 1747 , une instruction ${ }^{101}$ sur le bureau de vérification des hôpitaux a établi une procédure de contrôle. On y apprend que la finalité du bureau est de vérifier les états des hôpitaux pièce par pièce. Selon l'instruction, sont appelés pièces, les billets d'entrée, les certificats des aumôniers pour la mort des soldats et les certificats des chirurgiens-majors pour les convalescents hors d'état de servir. Le vérificateur doit s'assurer que les pièces sont jointes à l'état des hôpitaux ; s’il en manque une, il doit faire une note pour chaque état d'hôpital selon un modèle. Puis il passe à l'examen de chaque article de l'état qu'il pointe au vu des pièces ; en cas d'erreur, il ne pointe pas l'article mais indique l'erreur dans une note. Enfin, l'état de l'hôpital et les pièces sont remis au chef de bureau, qui vérifie la feuille de vérification. Le chef de bureau rend compte au premier

101 Ibid., "Instruction sur le travail du Bureau pour la vérification des États des d’hôpitaux », dans " Recueil de tout ce qui a été fait à l'occasion du nouvel arrangement des hôpitaux depuis le $1^{\text {er }}$ février 1746 au $1^{\text {er }}$ janvier 1749 », p. 103-107. 
commis des abus, et le premier commis reçoit alors les ordres du secrétaire d'État ayant le département de la Guerre pour les expéditions.

Si le bureau pour la vérification des états des hôpitaux existait avant 1747 , à partir de cette année-là on constate une augmentation régulière de ses effectifs. Ainsi, durant l'année 1746, huit commis composent le personnel du bureau, auxquels il faut ajouter un premier commis et un chef de bureau ${ }^{102}$. En 1757, le bureau des hôpitaux comprend dorénavant onze commis. À titre de comparaison, le secrétariat général à la Guerre est composé de quatorze bureaux comprenant en tout cent dix-neuf commis, ingénieurs ou peintres : la moyenne des commis par bureau est de huit et demi, ce qui classe alors le bureau des hôpitaux comme important en personnel ${ }^{103}$. Les commis ont des fonctions très spécialisées : il existe un commis de confiance qui reçoit quatre mille livres, deux commis vérificateurs ayant chacun deux mille livres, un commis tenant le registre des soldats décédés dans les hôpitaux recevant mille six cents livres, quatre commis aux écritures recevant chacun mille cinq cents livres, et un autre sans fonction particulière qui reçoit mille livres ${ }^{104}$.

Tous ces contrôles sur les officiers de santé ont au fil du temps façonné une pratique uniforme dans l'exercice de la médecine et de la chirurgie militaire. Un mémoire de 1770 atteste de cette réalité ; il prévoit deux mesures pour unifier le diagnostic des médecins, et spécialement ceux servant dans les hôpitaux ambulants.

L'auteur part d'un constat simple : le médecin civil traite son malade quotidiennement en prescrivant chaque jour une ordonnance suivant les symptômes. Il poursuit en remarquant que cette pratique ne peut être adoptée dans les hôpitaux militaires, du fait du grand nombre de malades dont chaque médecin à la charge. Selon l'auteur, les médecins militaires ont alors conçu une dizaine de formules simples ou composées pour s'en servir à la hâte, c'est pourquoi les jeunes médecins les apprennent avant de pratiquer eux-mêmes. Or, cette méthode n'est pas pratiquée dans les hôpitaux ambulants où la confusion règne parce qu' « à force de voir trop de malades il [le jeune médecin] ne voit pas la maladie ». L'auteur est sans nul doute médecin des hôpitaux militaires ${ }^{105}$. Il préconise d'appliquer la méthode des formules aux hôpitaux ambulants. Il souhaite aussi instaurer un Conseil de médecins praticiens, qui recevrait tous les mois de chaque médecin d'hôpital un journal des malades traités. Le Conseil rendrait alors des avis, surtout en cas de maladie épidémique, et en rendrait compte au ministre, lequel les transmettrait à l'intendant, qui les passerait au commissaire des guerres, qui les diffuserait aux médecins, chirurgiens et apothicaires ${ }^{106}$.

La proposition est reprise dans les ordonnances du 26 février $1777^{107}$ et du $1^{\text {er }}$ janvier $1780^{108}$, ainsi que dans le règlement du 18 mai $1788^{109}$. Ce règlement est le plus complet sur ce point puisqu'il instaure deux commissions : la première sous la dénomination de directoire des hôpitaux militaires, la seconde sous le nom de conseil de santé. Le directoire est chargé de la partie exécutive et de l'administration, le conseil de santé est chargé de la partie consultative pour tout ce qui est relatif à l'objet médical. Mais il est précisé « qu'agissant séparément en

102 SHD, Ya 25, Appointements des commis du bureau établi pour la vérification des états d’hôpitaux militaires et Frais de bureau de janvier à décembre 1746.

103 Ibid., États des bureaux de la guerre au premier février 1757.

104 Ibid., chemise de l'année 1759, extrait de la Décision de Monseigneur du 31 janvier 1759 pour l'arrangement et le traitement des bureaux de la guerre.

105 À propos des formules utilisées dans les hôpitaux militaires, il dit : " on donne de nous à toutes ces compositions ». L'emploi du « nous » semble indiquer son appartenance au personnel des hôpitaux militaires.

106 SHD, $\mathrm{A}^{2}$ 80, doc. 35, « Mémoire sur les hôpitaux militaires du 17 janvier 1770 ».

107 AD Nord, pl. 8448.

108 ISAMBERT, Recueil général..., op. cit., t. 26, p. 236-242.

109 Ibid., p. 569-573. 
ce qui les concerne, ces deux commissions puissent au besoin réunir leurs lumières et leurs soins dans les objets qui leur seront communs ».

Le titre deux du règlement est consacré exclusivement au Conseil de santé. Ce dernier est composé de huit membres en activité et de quatre membres honoraires, tous médecins et chirurgiens. Ces membres sont chargés de proposer les moyens les plus convenables à l'amélioration du service de santé. Ils éclairent aussi le directoire sur les moyens de perfectionner l'instruction des officiers de santé et sur leur avancement. Ce dernier point est à mettre en relation avec les fonctions du rapporteur du directoire, puisque ce dernier tient un registre de tous les officiers de santé « avec des notes de leurs talents et leurs services ».

Il ressort de la grande réformation entreprise par l'ordonnance de 1747, complétée par d'autres ordonnances ou règlements, qu'un contrôle hiérarchique des officiers de santé, doublé d'un contrôle budgétaire des hôpitaux militaires ${ }^{110}$, ont permis de rationaliser le statut des officiers de santé. Le résultat principal est d'avoir placé les officiers de santé sous l'étroite dépendance du secrétaire d'État ayant le département de la Guerre, lequel, par divers bureaux ou commissions, avait la mainmise sur la carrière des officiers de santé et même sur les aspects purement médicaux et chirurgicaux. Par ailleurs, cette centralisation décisionnelle a eu pour effet de priver le personnel de santé et les représentants locaux du pouvoir royal de la liberté qu'ils avaient avant 1747 ; elle a également unifié des techniques de gestion administrative du personnel en développant le bureau des hôpitaux, qui devait contrôler toutes les charges incombant au personnel hospitalier. Contraint de respecter une procédure méticuleuse, le personnel de santé a vu ses différents statuts s’unifier progressivement.

Renaud Limelette Université de Lille, Centre d’Histoire Judiciaire (UMR 8025) Faculté des sciences politiques, juridiques et sociales de Lille 1 Place Déliot, 59000 Lille renaud.limelette@univ-lille.fr

110 Pour aller plus loi, Renaud LIMELETTE, « La gouvernance du service de santé des hôpitaux militaires de la réforme de 1747 à 1789 », op. cit., note 22. 